\title{
A Review of the Epidemiology, Pathophysiology, and Efficacy of Anti-diabetic Drugs Used in the Treatment of Nonalcoholic Fatty Liver Disease
}

\author{
Paul P. Manka ${ }^{1} \cdot$ Eda Kaya $^{1} \cdot$ Ali Canbay ${ }^{1} \cdot$ Wing-Kin Syn ${ }^{2,3,4}$
}

Accepted: 22 July 2021 / Published online: 19 August 2021

(c) The Author(s) 2021

\begin{abstract}
In recent years, epidemiological studies have consistently demonstrated that the coexistence of nonalcoholic fatty liver disease (NAFLD) and type 2 diabetes mellitus (T2DM) is strongly associated with increased mortality and morbidity related to hepatic- and extrahepatic causes. Indeed, compared with the general population, patients with T2DM are more likely to be diagnosed with more severe forms of NAFLD (i.e., nonalcoholic steatohepatitis (NASH) with liver fibrosis). There is an ongoing debate whether NALFD is a consequence of diabetes or whether NAFLD is simply a component and manifestation of the metabolic syndrome, since liver fat (steatosis) and even more advanced stages of liver fibrosis can occur in the absence of diabetes. Nevertheless, insulin resistance is a key component of the mechanism of NAFLD development; furthermore, therapies that lower blood glucose concentrations also appear to be effective in the treatment of NAFLD. Here, we will discuss the pathophysiological and epidemiological associations between NAFLD and T2DM. We will also review currently available anti-diabetic agents with their regard to their efficacy of NAFLD/NASH treatment.
\end{abstract}

Keywords NAFLD $\cdot$ NASH $\cdot$ Diabetes $\cdot$ Fibrosis

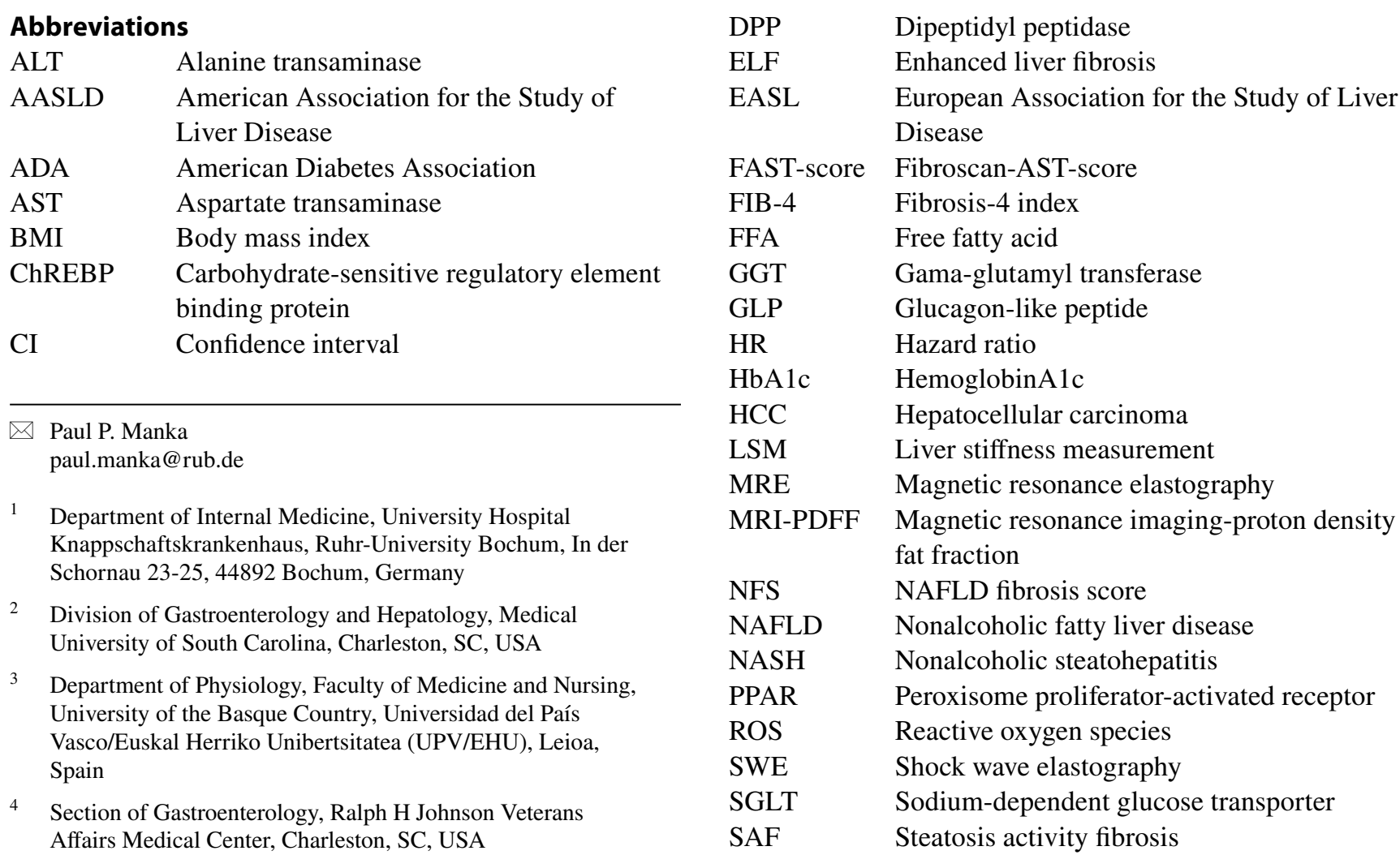




$\begin{array}{ll}\text { SREBP } & \text { Sterol sensitive regulatory element binding } \\ & \text { protein } \\ \text { TNF } & \text { Tumor necrosis factor } \\ \text { T2DM } & \text { Type 2 diabetes mellitus } \\ \text { UNOS } & \text { United Network for Organ Sharing } \\ \text { VLDL } & \text { Very low density lipoproteins } \\ \text { VCTE } & \text { Vibration-controlled transient elastography }\end{array}$

\section{Introduction}

Nonalcoholic fatty liver disease (NAFLD) encompasses a spectrum of histopathologic conditions, ranging from simple steatosis to nonalcoholic steatohepatitis (NASH), with or without liver fibrosis, cirrhosis and hepatocellular carcinoma (HCC). It is defined as excess hepatic fat accumulation (>5\%) in hepatocytes, assessed by either imaging or histology. Furthermore, secondary causes of excess liver fat, including significant or excess alcohol consumption $(\geq 30 \mathrm{~g} /$ day in men and $/ \geq 20 \mathrm{~g} /$ day in women) must be excluded $[1,2]$.

Affecting nearly $25 \%$ of the world population, NAFLD can be regarded as the world's most common chronic liver disease [3]. Unfortunately, the burden of NALFD and its progressive form NASH is predicted to increase. A recently published modeling study, applied to China, France, Germany, Italy, Japan, Spain, the UK, and the USA, has predicted a significant increase in the prevalence of NAFLD, with more than a doubling of cases with advanced liver disease and liver-related mortality in the coming years. Of concern, this increase in NAFLD prevalence parallels the predicted increase in prevalence of obesity and type 2 diabetes mellitus (T2DM) [4]. Data from the United Network for Organ Sharing (UNOS) confirms this; in 2017, there were more patients with NAFLD on the liver transplant waiting list than there were patients with chronic viral hepatitis C [5].

Herein, we aim to highlight the relationship between NAFLD and T2DM, considering epidemiological, pathophysiological, and clinical components. We will also discuss potential therapeutic strategies that may be applied to managing both conditions.

\section{Epidemiology}

NAFLD represents a substantial clinical burden affecting $25 \%$ of the world population; a recently published metaanalysis with data obtained from 20 different countries reported that NAFLD prevalence is twice as high among those with T2DM compared with the general population. Among Europeans, the prevalence of NAFLD among those with T2DM is nearly three times higher [6]. Conversely, a recent meta-analysis revealed that patients with NAFLD are at a 2.2-fold increased risk of developing incident T2DM [7].

In addition to the high prevalence of NAFLD among those with T2DM, the presence of NAFLD appears to have a marked impact on clinical outcomes. In a longitudinal study with 150-months follow-up in patients with biopsyproven NAFLD, having both NAFLD and T2DM was associated with a doubling of all-cause and liver-related mortality (Hazard ratio (HR): 2.09 [95\% Confidence interval (CI): 1.39-3.14] and 2.19 [95\%CI: 1.00-4.81], respectively) [8]. While the estimated prevalence of NASH in the general population is $3-5 \%$ [3], a recent meta-analysis found that the global prevalence of NASH among the diabetic population was $37.3 \%$ (95\% CI 24.7-50.0\%) and that a significantly high proportion of those with T2DM and NAFLD had advanced NASH fibrosis (17\%). The global prevalence of advanced fibrosis among patients with T2DM was estimated as 4.8\% [6]. This is of major clinical importance since fibrosis stage is regarded as the most important predictor of liver-related mortality [9]. Particular attention should be paid to "lean NAFLD" patients who represent up to one-fifth of the NAFLD population [10], who were at increased risk of developing T2DM [11, 12]. Furthermore, T2DM was the most important risk factor for NAFLD progression in this cohort [13].

Diabetes is also significant risk factor for the development of hepatocellular carcinoma (HCC). One large study of 18 million patients revealed that diabetes is the strongest independent predictor of $\mathrm{HCC}$ or cirrhosis (HR 2.3, 95\% CI 1.9-2.78) [14], which is supported by a recent study of 354 subjects with NASH cirrhosis that confirmed that the risk of HCC development is significantly increased among those with T2DM (HR 4.2; 95\% CI 1.2-14.2) [15].

\section{Pathophysiology and Disease Progression}

Accurate predictions of the probability or rate of NAFLD progression cannot be made at present. Known risk factors for NAFLD progression include genetic factors, such as the Ile148Met substitution of the adiponutrin gene termed PNPLA3, elevated body mass index (BMI), and comorbid factors, particularly the presence or absence of T2DM. Alternative concepts regard NAFLD not as a consequence, but rather a cause of T2DM, since the liver releases proinflammatory hepatokines, which may accelerate the development of diabetes [16].

Due to the complexities of the pathogenesis of $\mathrm{NASH}$, the understanding of its pathogenic mechanisms among individual patients with NAFLD/NASH remains 
incomplete. Fat accumulation in liver parenchymal cells occurs when the synthesis and supply of neutral fats exceed hepatic clearance. Numerous factors, including insulin resistance, endotoxins, proinflammatory cytokines, oxidative stress, alterations of mitochondria, apoptotic processes, and genetic factors, contribute to NAFLD pathogenesis. Constitutional factors such as age and genetic predisposition, as well as clinical risk factors such as increased BMI, especially with visceral obesity, physical inactivity, increased caloric intake, and the presence of insulin resistance or type 2 diabetes, are associated with the development and/or progression of NAFLD. Recent studies further suggest that the composition of the gut microbiome and intake of specific foods such as fructose may also promote the development and/or progression of NAFLD [17]. Insulin resistance, especially in adipose tissue and skeletal muscle, is fundamental to the pathogenesis of NAFLD. NAFLD is therefore usually regarded as a hepatic manifestation of the metabolic syndrome and diabetes. In healthy individuals, insulin inhibits hormonesensitive lipase in adipose tissue, inhibiting triglyceride hydrolysis and free fatty acid (FFA) release [18]. In those with NAFLD, increased visceral adipocyte mass and the disinhibited activity of hormone-sensitive lipase in insulin resistance increase triglyceride hydrolysis, which increase plasma levels of FFAs, especially in the portal venous blood. Consequently, uptake of FFA into hepatocytes is increased. Furthermore, skeletal muscle insulin resistance also indirectly increases lipid uptake into hepatocytes as a consequence of reduced muscle glucose uptake [19, 20]. Unlike adipose tissue and skeletal muscle, insulin resistance is only partial in the liver of patients with NAFLD/ NASH. On the one hand, glucose regulation is dysregulated in a steatotic liver (i.e., insulin resistant) [21], and the liver does not respond to regulatory signals; on the other hand, hepatic lipogenesis remains insulin sensitive even under insulin-resistant states, increasing lipogenesis, with resultant accumulation of liver triglycerides. In animal models, both insulin and glucose independently regulate de novo lipogenesis (DNL) via activation of the steroland carbohydrate-sensitive regulatory element binding proteins, SREBP-1c and ChREBP, two central genes that activate hepatic lipogenesis [22, 23]. Chronic hyperinsulinemia also decreases apolipoprotein B100 synthesis and thus very low-density lipoproteins (VLDL)-associated lipid export from liver cells. Therefore, hyperinsulinemia increases hepatic triglyceride synthesis with concomitant inhibition of triglyceride secretion as VLDL (steatosis) [24]. Furthermore, FFAs in the liver enhance lipid peroxidation generate highly reactive oxygen species (ROS) and stimulate the expression of proinflammatory cytokines such as tumor necrosis factor (TNF)- $\alpha$, which enhances the necroinflammatory processes and liver fibrosis (steatohepatitis). During DNL, toxic metabolites such as diacylglycerol and ceramides can also induce insulin resistance, thereby creating a positive feedback loop wherein insulin resistance stimulates hepatic DNL, and hepatic DNL in turn promotes insulin resistance [25].

\section{Clinical Assessment}

The complexity of pathogenic mechanisms and heterogeneity of NAFLD complicate accurate prediction of NAFLD prognosis. In general, about one-third of those with simple steatosis will progress to NASH and about one-third of those with NASH will develop significant liver fibrosis or cirrhosis.

Screening the general population for NAFLD is currently not recommended [2]. Even among those with NAFLD, only patients with an increased risk of developing complications need further evaluation and/or regular follow-up since only a minority of those with NAFLD will experience a severe clinical event [8]. Therefore, the evaluation and risk stratification should be focused on identifying those considered at high risk of progression to cirrhosis and cirrhosis-associated complications. Indeed, the European Association for the Study of Liver Disease (EASL) recommends a routine, non-invasive initial evaluation for individuals with the metabolic syndrome, obesity, and T2DM [1] and the American Diabetes Association (ADA) guidance recommends a yearly assessment of liver transaminases in those with T2DM [26]. Screening patients using transaminases alone, however, is inaccurate as it will likely miss a significant proportion of those with significant or advanced liver fibrosis (i.e., those with F2 or greater fibrosis stage) since $25 \%$ of patients with NAFLD, and $19 \%$ of those with NASH will have normal transaminases [27]. At this time, the American Association for the Study of Liver Disease (AASLD) does not recommend screening for NAFLD [2], although a recent costutility analysis by investigators of NASHNET found that screening among those with T2DM is cost-effective [28].

As NAFLD is a component of the metabolic syndrome, and as such, a multisystem disorder, there is agreement that management of NAFLD should involve a multidisciplinary approach. Such an interdisciplinary team would likely consist of hepatologists, diabetologists, cardiologists, and nutritional doctors, as well as dietitians, exercise physiologists, and psychologists [29, 30]. Indeed, studies comparing models of care (i.e., care provided by a multidisciplinary team versus single providers) in patients with heart failure have consistently demonstrated superior outcomes using a multidisciplinary approach, including a reduction of cardiovascular risk $[29,30]$. The high prevalence of NAFLD, however, makes it costly and impractical to provide such 
multidisciplinary service for all patients and at all levels of service provision. One proposed model of care would be for primary care physicians and/or diabetologists to refer those identified at highest risk of NAFLD progression using established guidelines. Secondary or tertiary centers with greater resources should ideally manage these high-risk patients using a multidisciplinary approach [31,32] (Fig. 1).

The preferred first-line diagnostic methods involve the use of non-invasive scoring systems based on widely available serum biomarkers. The Fibrosis-4 index (FIB-4) and NAFLD fibrosis score (NFS) are well-validated and recommended by several guidelines $[1,2,33]$. Due to its simple calculation, FIB-4 seems to be best adapted to daily practice [33]. The capability of those tests lies in their ability to exclude advanced fibrosis (i.e., excellent negative predictive value), rather than in diagnosing early disease, a desirable characteristic among those with T2DM [34,
35]. Specifically, a FIB-4 cutoff of $<1.3$ suggests a low risk of advanced fibrosis and a score $>2.67$ suggests high risk of advanced fibrosis. Patients with an indeterminate score (i.e. $\geq 1.3$ but $<2.67$ ) or a score $>2.67$ are recommended to be further evaluated with a second modality (e.g., NFS, the enhanced liver fibrosis (ELF) score, or vibration-controlled transient elastography (VCTE)) [32]. For these individuals, undergoing FIB4 followed by VCTE appears to be the most cost-effective strategy for disease stratification [28, 36, 37]. This stepwise approach reduces the number of unnecessary liver biopsies [38, 39], and costs. The FibroScan-AST (FAST) ${ }^{\mathrm{TM}}$ score (alanine transaminase/aspartate transaminase ratio combined with fibroscan measurement) improves identification of those at the highest risk of disease progression (i.e., those with a NAFLD fibrosis score $\geq 4$ and fibrosis stage $\geq 2$ ) [40]. Apart from VCTE, magnetic resonance elastography

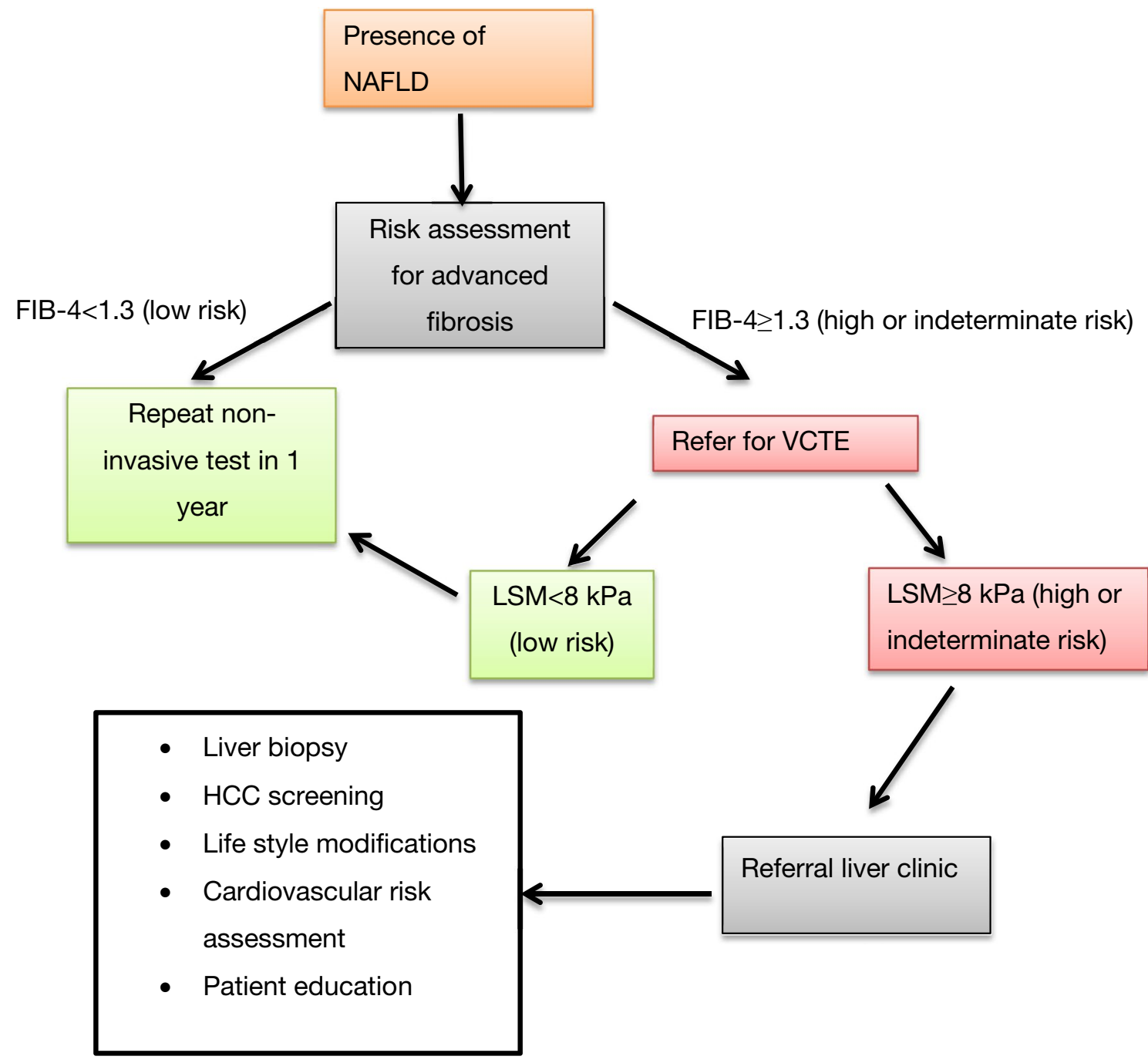

Fig. 1 Diagnostic approach in NAFLD patients with T2DM. NAFLD Nonalcoholic fatty liver disease, T2DM Type 2 diabetes mellitus, FIB-4 Fibrosis-4 index, VCTE Vibration-controlled transient elastography, LSM Liver stiffness measurement, HCC Hepatocellular carcinoma 
(MRE) or 2D-shock wave elastography (SWE) could also be considered as the second modality [41, 42], but are costly and are currently not widely available [43].

\section{Therapeutics}

Despite the significant burden of the disease, there is no current approved pharmacological therapy for NAFLD $[1,2,44]$.

\section{Lifestyle Modification}

Lifestyle modifications targeting weight loss remain the cornerstone of treatment, which also constitutes the backbone of diabetes management [44]. Indeed, a body weight reduction of 5\% decreases liver fat content, whereas weight loss of $7-10 \%$ can even regress NASH and/or liver fibrosis [45]. In the future, digital education will likely assume increasing importance in the management of NAFLD. In a recently conducted clinical trial, 12 weeks of diet and exercise supported by digital education reduced body weight by $\sim 9 \%$ and resolved NAFLD in $30 \%$ of participants with NAFLD and T2DM [46]. A major limitation of all lifestyle modification programs is non-compliance and the inability to sustain weight loss beyond the prescribed program. Indeed, fewer than half of the patients undergoing lifestyle intervention programs achieved the targeted weight loss, and only $25 \%$ of the patients managed to sustain the weight loss of $>5 \%$ [47]. As such, there is increased efforts in developing new pharmacological treatment for NAFLD.
Although there is currently no approved pharmacological therapy specifically for NAFLD/NASH, several already approved anti-diabetic agents have shown promise in NAFLD/NASH. We will discuss these below.

\section{Pharmacological Approach}

While multiple NASH/anti-fibrotic agents are being evaluated in phase 2 and phase 3 trials [48], several drugs already approved for the treatment of T2DM are beneficial for NAFLD/NASH. Specifically, we will summarize recent randomized controlled trials that evaluated (or are evaluating) anti-diabetic agents in the treatment of NAFLD/NASH in Tables 1 and 2.

\section{Metformin}

In diabetology, cardiovascular safety studies have led to an overall paradigm shift in the therapy of diabetes. Metformin is now listed in the current European recommendations [49] as the only first-line agent for diabetic patients without cardiovascular complications. In the presence of cardiovascular disease, sodium-dependent glucose transporter (SGLT)-2 inhibitors and stable glucagon-like peptide (GLP)1 analogs are preferred. Most of the data on anti-diabetic drug therapy in NAFLD are derived from studies of metformin. The beneficial effect of metformin is mainly based on reducing the risk of HCC [50]. In a sizeable multivariable regression analysis [51], diabetes was associated with a 1.35-fold increased risk of HCC compared with the control group. Analysis of associated medications showed that metformin, in particular, was associated with a $30 \%$ lower risk of HCC, as was reported in cohort analyses of other

Table 1 Recent biopsy-proven randomized controlled trials including anti-diabetic agents

\begin{tabular}{|c|c|c|c|}
\hline Drug group & Drug name & Study characteristics & Outcome \\
\hline DPP4 inhibitor & Sitagliptin [56] & $\begin{array}{l}100 \mathrm{mg} / \text { day dose of sitagliptin versus placebo } \\
24 \text { weeks of follow-up }\end{array}$ & $\begin{array}{l}\text { No significant } \\
\text { improvement in } \\
\text { fibrosis or NAFLD } \\
\text { fibrosis score }\end{array}$ \\
\hline \multirow[t]{2}{*}{ GLP1 agonist } & Liraglutide [59] & $\begin{array}{l}1.8 \mathrm{mg} / \text { day dose of Liraglutide versus placebo } \\
48 \text { weeks of follow-up }\end{array}$ & NASH resolution \\
\hline & Semaglutide [60] & $\begin{array}{l}0.1,0.2,0.4 \mathrm{mg} \text { daily versus placebo } \\
72 \text { weeks of follow-up }\end{array}$ & $\begin{array}{l}\text { NASH resolution } \\
\text { No significant } \\
\text { change in liver } \\
\text { fibrosis between } \\
\text { groups }\end{array}$ \\
\hline SGLT-2 inhibitor & - & - & - \\
\hline PPAR agonist & Pioglitazone [74] & $\begin{array}{l}45 \mathrm{mg} / \text { day dose of pioglitazone versus placebo supported } \\
\text { with low-caloric diet } \\
18 \text { months of follow-up }\end{array}$ & NASH resolution \\
\hline
\end{tabular}

DPP Dipeptidyl peptidase, NAFLD Nonalcoholic fatty liver disease, GLP Glucagon-like peptide, NASH Nonalcoholic steatohepatitis, SGLT Sodium-dependent glucose transporter, PPAR Peroxisome proliferator-activated receptor 


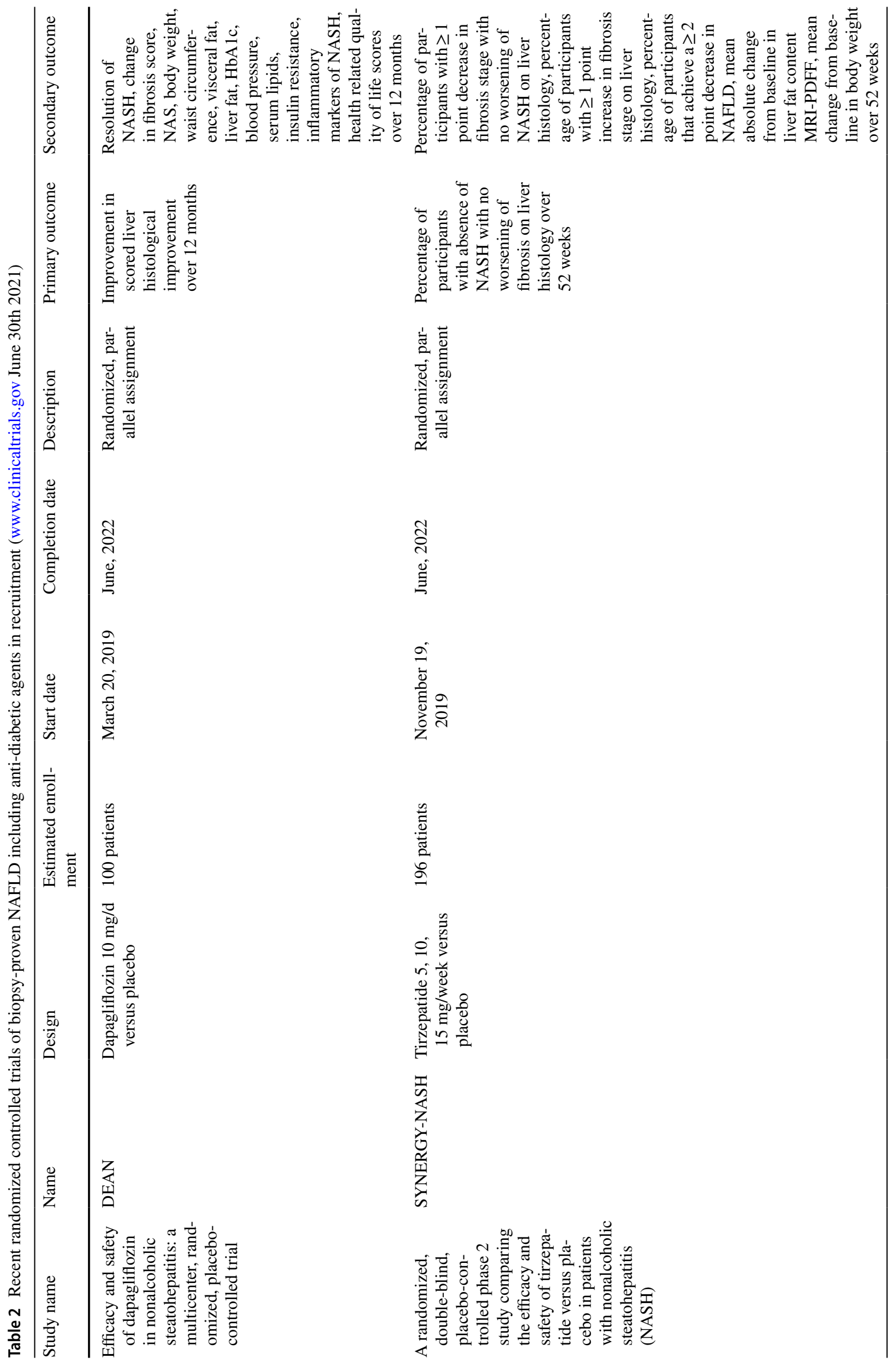




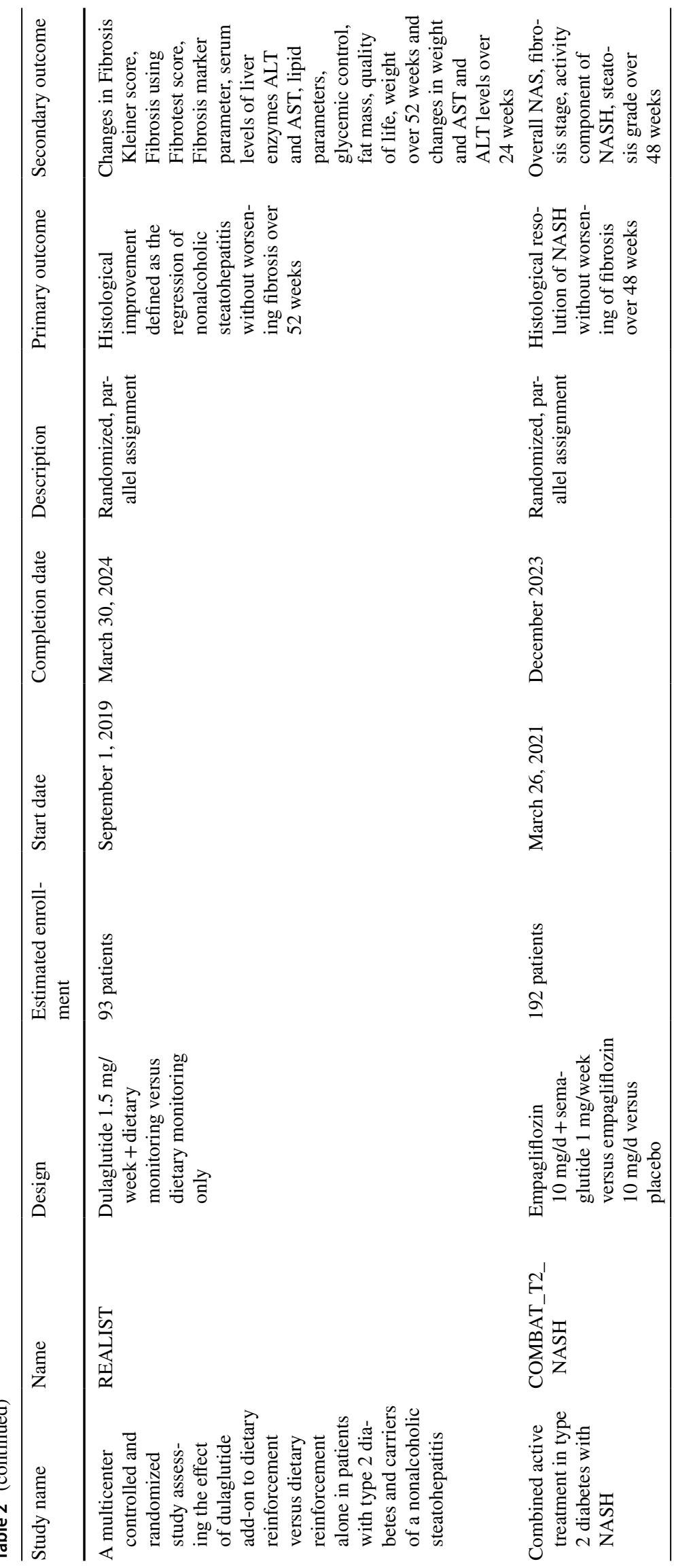




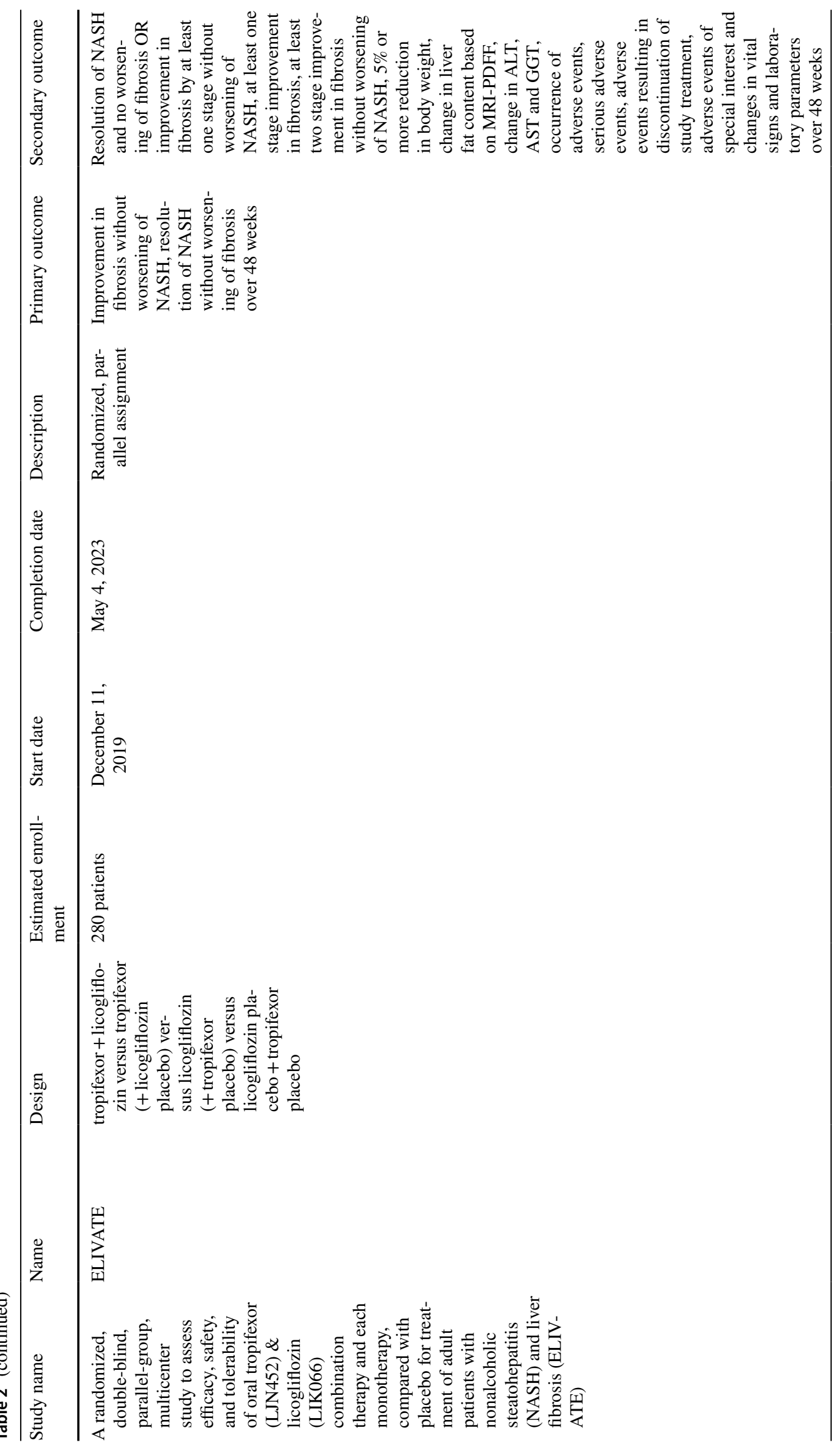




$$
\Pi
$$


tumor entities. Sulfonylureas were neutral, whereas insulin therapy increased the risk of HCC 1.6-fold. Lipid-lowering treatment, i.e., statin use, had the most pronounced effect on HCC risk, with a $35 \%$ reduction. Nevertheless, metformin does not affect steatosis per se [50]. Indeed, a meta-analysis that included randomized controlled trials revealed that metformin did not significantly impact steatosis, ballooning, and fibrosis. Lobular inflammation was actually increased in patients taking metformin [52]. Therefore, the beneficial effect of metformin in NAFLD therapy remains uncertain.

\section{Dipeptidyl Peptidase (DPP)4 Inhibitors}

Since dipeptidyl peptidase (DPP) 4 is the primary metabolic enzyme for GLP1, an enteroendocrine hormone with salutary effects on insulin release, motility, and appetite [53], its inhibition increases the half-life $\left(t_{1 / 2}\right)$ of GLP1 and related peptides in the circulation. Accumulating data suggest that sitagliptin has no beneficial effect on hepatic outcomes in NAFLD patients, although some controversial results showed a possible beneficial effect of sitagliptin in NASH improvement $[54,55]$. Nonetheless, no significant improvement was observed in terms of NAFLD fibrosis score and fibrosis stage in a biopsy-proven randomized controlled study [56]. Though its only advantage remains in its good tolerability and safety, this is insufficient justification for its use as a management option in NAFLD [54].

\section{GLP1 Receptor Agonists}

Stable GLP1 receptor agonist analogs significantly decrease body weight and food intake by reducing gastric emptying and possibly by affecting central satiety centers in addition to their hypoglycemic effects [57]. According to a recent meta-analysis, GLP1 receptor agonists are a new therapeutic option for resolving NASH without worsening fibrosis [58]. In a placebo-controlled phase 2 study, liraglutide resolved histological NASH compared with placebo (39\% vs. $9 \%, P=0.019)$ though a significant change in the mean NAFLD activity score was not observed [59]. Semaglutide demonstrated similar results: patients with biopsy-proven NASH receiving semaglutide $0.4 \mathrm{mg}$ daily resolved NASH at rates higher than those receiving placebo (59\% vs. $17 \%$, $P<0.001)$. Though fibrosis stage improvement was not significantly different [60]. Exenatide reduced hepatic fat [61]. A combination of exenatide and dapagliflozin, a SGLT2 inhibitor, ameliorated hepatic steatosis and fibrosis markers compared with dapagliflozin and placebo and exenatide alone, suggesting that combination therapies may be beneficial and are worthy of further investigation [62].

\section{SGLT2 Inhibitors}

SGLT2, expressed in the renal proximal tubule, is the primary mechanism of renal capture of filtered glucose. Its inhibition lowers the threshold for glycosuria with resultant improvement in glycemic control while facilitating negative caloric balance [63].

In randomized clinical trials, SGLT2 inhibitors mostly reduced hepatic fat content $[64,65]$. In line with accumulating data, SGLT2 inhibitors were proposed as valuable agents in the diabetic NAFLD population in order to regulate blood glucose and improve hepatic fat content and fibrosis [66]. Furthermore, beneficial effects in reducing cardiovascular risk and nephropathy progression were also proposed $[67,68]$. A recent meta-analysis included randomized controlled trials conducted in Asian populations demonstrated improved anthropometric measurements, liver enzymes, serum lipids, glycemic control, inflammatory markers, and serum biomarkers predicting liver fibrosis [69]. From those SGLT2 inhibitors, dapagliflozin and empagliflozin significantly reduced the hepatic fat content assessed by magnetic resonance imaging-proton density fat fraction (MRI-PDFF) [70, 71]. In a biopsy-proven NASH cohort with T2DM, empagliflozin effectively improved liver steatosis, ballooning and fibrosis, and NASH resolution in half of the patients in a follow-up of 6 months [72].

Peroxisome Proliferator-Activated Receptor (PPAR) Agonists The peroxisome proliferator-activated receptor (PPAR) $\alpha$ is a nuclear receptor linked to inflammation, insulin sensitization, and lipid metabolism [73]. Its agonists have met with some success in the treatment of metabolic disease. In this class of drugs, though pioglitazone accelerated NASH resolution and improved advanced fibrosis [74, 75], due to unfavorable adverse effects such as edema, bone fracture, cardiovascular disease development, and weight gain, its use in NAFLD treatment has been withdrawn [76, 77]. Finally, a randomized controlled, double-blind trial reported positive effects of saroglitozar in the improvement of hepatic fat content diagnosed by MRI-PDFF, insulin resistance, alanine transaminase levels, and serum lipid profiles in NAFLD patients [78].

\section{Conclusion}

- This review primarily highlighted the close association between NAFLD and T2DM and the clinical approach to patients with these two conditions. The success of anti-diabetic drugs with NASH combined with the growing associational and pathophysiologic links between metabolic liver disease and the metabolic syndrome indicates the bidirectional nature of the relationship between 
these entities. Although there is no approved therapy for NAFLD, therapies used to treat diabetes seem to be a logical solution for NAFLD management. As the pathogenesis of NAFLD becomes better understood, treatments aimed at the unique factors involved in NAFLD pathogenesis should show even better efficacy for NAFLD treatment than do the currently used anti-diabetic therapies.

Author's contribution PM and EK were involved in drafting of the manuscript (PM and EK contributed equally to this manuscript), and WKS and AC were involved in critical revision.

Funding Open Access funding enabled and organized by Projekt DEAL. The authors received no financial support to produce this manuscript.

\section{Declarations}

Conflict of interest The authors declare that they have no conflict of interest.

Open Access This article is licensed under a Creative Commons Attribution-NonCommercial 4.0 International License, which permits any non-commercial use, sharing, adaptation, distribution and reproduction in any medium or format, as long as you give appropriate credit to the original author(s) and the source, provide a link to the Creative Commons licence, and indicate if changes were made. The images or other third party material in this article are included in the article's Creative Commons licence, unless indicated otherwise in a credit line to the material. If material is not included in the article's Creative Commons licence and your intended use is not permitted by statutory regulation or exceeds the permitted use, you will need to obtain permission directly from the copyright holder. To view a copy of this licence, visit http://creativecommons.org/licenses/by-nc/4.0/.

\section{References}

1. European Association for the Study of the Liver (EASL); European Association for the Study of Diabetes (EASD); European Association for the Study of Obesity (EASO). EASL-EASDEASO Clinical Practice Guidelines for the management of nonalcoholic fatty liver disease. J Hepatol. 2016;64:1388-402.

2. Chalasani N, Younossi Z, Lavine JE, Charlton M, Cusi K, Rinella M, Harrison SA, Brunt EM, Sanyal AJ. The diagnosis and management of non-alcoholic fatty liver disease: practice guidance from the American Association for the Study of Liver Diseases. Hepatology. 2018;67:328-357.

3. Younossi Z, Tacke F, Arrese M, Chander Sharma B, Mostafa I, Bugianesi E, Wai-Sun Wong V, Yilmaz Y, George J, Fan J, Vos MB. Global perspectives on nonalcoholic fatty liver disease and nonalcoholic steatohepatitis. Hepatology. 2019;69:2672-2682.

4. Estes C, Anstee QM, Arias-Loste MT, Bantel H, Bellentani S, Caballeria J, Colombo M, Craxi A, Crespo J, Day CP, Eguchi Y, Geier A, Kondili LA, Kroy DC, Lazarus JV, Loomba R, Manns MP, Marchesini G, Nakajima A, Negro F, Petta S, Ratziu V, Romero-Gomez M, Sanyal A, Schattenberg JM, Tacke F, Tanaka J, Trautwein C, Wei L, Zeuzem S, Razavi H. Modeling NAFLD disease burden in China, France, Germany, Italy, Japan, Spain, United Kingdom, and United States for the period 2016-2030. J Hepatol. 2018;69:896-904.

5. Goldberg D, Ditah IC, Saeian K, Lalehzari M, Aronsohn A, Gorospe EC, Charlton M. Changes in the prevalence of hepatitis $\mathrm{C}$ virus infection, nonalcoholic steatohepatitis, and alcoholic liver disease among patients with cirrhosis or liver failure on the waitlist for liver transplantation. Gastroenterology. 2017;152:10901099.e1.

6. Younossi ZM, Golabi P, de Avila L, Paik JM, Srishord M, Fukui N, Qiu Y, Burns L, Afendy A, Nader F. The global epidemiology of NAFLD and NASH in patients with type 2 diabetes: a systematic review and meta-analysis. J Hepatol. 2019;71:793-801.

7. Mantovani A, Petracca G, Beatrice G, Tilg H, Byrne CD, Targher G. Non-alcoholic fatty liver disease and risk of incident diabetes mellitus: an updated meta-analysis of 501022 adult individuals. Gut. 2021;70:962-969.

8. Stepanova M, Rafiq N, Makhlouf $\mathrm{H}$ et al. Predictors of all-cause mortality and liver-related mortality in patients with non-alcoholic fatty liver disease (NAFLD). Dig Dis Sci. 2013;58:30173023. https://doi.org/10.1007/s10620-013-2743-5.

9. Ekstedt M, Hagström H, Nasr P, Fredrikson M, Stål P, Kechagias $\mathrm{S}$ et al. Fibrosis stage is the strongest predictor for diseasespecific mortality in NAFLD after up to 33 years of follow-up. Hepatology. 2015;61:1547-1554.

10. Ye Q, Zou B, Yeo YH, Li J, Huang DQ, Wu Y, Yang H, Liu C, Kam LY, Tan XXE, Chien N, Trinh S, Henry L, Stave CD, Hosaka T, Cheung RC, Nguyen MH. Global prevalence, incidence, and outcomes of non-obese or lean non-alcoholic fatty liver disease: a systematic review and meta-analysis. Lancet Gastroenterol Hepatol. 2020;5:739-752.

11. Wei L, Cheng X, Luo Y, Yang R, Lei Z, Jiang H, Chen L. Lean non-alcoholic fatty liver disease and risk of incident diabetes in a euglycaemic population undergoing health check-ups: A cohort study. Diabetes Metab. 2021;47:101200.

12. Sinn DH, Kang D, Cho SJ, Paik SW, Guallar E, Cho J, Gwak GY. Lean non-alcoholic fatty liver disease and development of diabetes: a cohort study. Eur J Endocrinol. 2019;181:185-192.

13. Park H, Yoon EL, Cho S, Jun DW, Nah EH. Diabetes is the strongest risk factor of hepatic fibrosis in lean patients with nonalcoholic fatty liver disease. Gut. 2021:gutjnl-2021-325102.

14. Alexander M, Loomis AK, van der Lei J et al. Risks and clinical predictors of cirrhosis and hepatocellular carcinoma diagnoses in adults with diagnosed NAFLD: real-world study of 18 million patients in four European cohorts. BMC Med. 2019;17:95.

15. Yang JD, Ahmed F, Mara KC, Addissie BD, Allen AM, Gores GJ, Roberts LR. Diabetes is associated with increased risk of hepatocellular carcinoma in patients with cirrhosis from nonalcoholic fatty liver disease. Hepatology. 2020;71:907-916.

16. Meex RCR, Watt MJ. Hepatokines: linking non-alcoholic fatty liver disease and insulin resistance. Nat Rev Endocrinol. 2017;13:509-520.

17. Friedman SL, Neuschwander-Tetri BA, Rinella M, Sanyal AJ. Mechanisms of NAFLD development and therapeutic strategies. Nat Med. 2018;24:908-922.

18. Zhao J, Wu Y, Rong X, Zheng C, Guo J. Anti-lipolysis induced by insulin in diverse pathophysiologic conditions of adipose tissue. Diabetes Metab Syndr Obes. 2020;11:1575-1585.

19. Jelenik T, Kaul K, Séquaris G, Flögel U, Phielix E, Kotzka J, Knebel B, Fahlbusch P, Hörbelt T, Lehr S, Reinbeck AL, Müller-Wieland D, Esposito I, Shulman GI, Szendroedi J, Roden M. Mechanisms of insulin resistance in primary and secondary nonalcoholic fatty liver. Diabetes. 2017;66:2241-2253.

20. Finck BN. Targeting metabolism, insulin resistance, and diabetes to treat nonalcoholic steatohepatitis. Diabetes. 2018;67:2485-2493. 
21. Brown MS, Goldstein JL. Selective versus total insulin resistance: a pathogenic paradox. Cell Metab. 2008;7:95-96.

22. Azzout-Marniche D, Bécard D, Guichard C, Foretz M, Ferré P, Foufelle F. Insulin effects on sterol regulatory-element-binding protein-1c (SREBP-1c) transcriptional activity in rat hepatocytes. Biochem J. 2000;350 Pt 2:389-393.

23. Browning JD, Horton JD. Molecular mediators of hepatic steatosis and liver injury. J Clin Invest. 2004;114:147-152.

24. Dashti N, Williams DL, Alaupovic P. Effects of oleate and insulin on the production rates and cellular mRNA concentrations of apolipoproteins in HepG2 cells. J Lipid Res. 1989;30:1365-1373.

25. Smith GI, Shankaran M, Yoshino M, Schweitzer GG, Chondronikola M, Beals JW, Okunade AL, Patterson BW, Nyangau E, Field T, Sirlin CB, Talukdar S, Hellerstein MK, Klein S. Insulin resistance drives hepatic de novo lipogenesis in non-alcoholic fatty liver disease. J Clin Invest. 2020;130:1453-1460.

26. American Diabetes Association. 4. Comprehensive medical evaluation and assessment of comorbidities: Standards of Medical Care in Diabetes-2020. Diabetes Care 2020;43:S37-47.

27. Ma X, Liu S, Zhang J, Dong M, Wang Y, Wang M, Xin Y. Proportion of NAFLD patients with normal ALT value in overall NAFLD patients: a systematic review and meta-analysis. BMC Gastroenterol. 2020;20:10. https://doi.org/10.1186/ s12876-020-1165-z.

28. Noureddin M, Jones C, Alkhouri N, Gomez EV, Dieterich DT, Rinella ME; NASHNET. Screening for nonalcoholic fatty liver disease in persons with type 2 diabetes in the United States is cost-effective: a comprehensive cost-utility analysis. Gastroenterology. 2020;159:1985-7.e4.

29. Ruissen MM, Mak AL, Beuers U, Tushuizen ME, Holleboom AG. Non-alcoholic fatty liver disease: a multidisciplinary approach towards a cardiometabolic liver disease. Eur J Endocrinol. 2020;183:R57-R73.

30. Cobbold JFL, Raveendran S, Peake CM, Anstee QM, Yee MS, Thursz MR. Piloting a multidisciplinary clinic for the management of non-alcoholic fatty liver disease: initial 5-year experience. Frontline Gastroenterol. 2013;4:263-269.

31. Budd J, Cusi K. Nonalcoholic fatty liver disease: What does the primary care physician need to know? Am J Med. 2020;133:536-543.

32. Vieira Barbosa J, Lai M. Nonalcoholic fatty liver disease screening in type 2 diabetes mellitus patients in the primary care setting. Hepatol Commun. 2020;5:158-167.

33. Eslam M, Sarin SK, Wong VW, Fan JG, Kawaguchi T, Ahn SH, Zheng MH, Shiha G, Yilmaz Y, Gani R, Alam S, Dan YY, Kao JH, Hamid S, Cua IH, Chan WK, Payawal D, Tan SS, Tanwandee T, Adams LA, Kumar M, Omata M, George J. The Asian Pacific Association for the Study of the Liver clinical practice guidelines for the diagnosis and management of metabolic associated fatty liver disease. Hepatol Int. 2020;14:889-919.

34. Alkayyali T, Qutranji L, Kaya E, Bakir A, Yilmaz Y. Clinical utility of non-invasive scores in assessing advanced hepatic fibrosis in patients with type 2 diabetes mellitus: a study in biopsy-proven non-alcoholic fatty liver disease. Acta Diabetol. 2020;57:613-618.

35. Kaya E, Bakir A, Kani HT, Demirtas CO, Keklikkiran C, Yilmaz Y. Simple noninvasive scores are clinically useful to exclude, not predict, advanced fibrosis: a study in turkish patients with biopsy-proven nonalcoholic fatty liver disease. Gut Liver. 2020;14:486-491.

36. Younossi ZM, Corey KE, Alkhouri N et al. Clinical assessment for high-risk patients with non-alcoholic fatty liver disease in primary care and diabetology practices. Aliment Pharmacol Ther. 2020;52:513-526.

37. Younossi ZM, Pham H, Felix S, Stepanova M, Jeffers T, Younossi E, Allawi H, Lam B, Cable R, Afendy M, Younoszai Z,
Afendy A, Rafiq N, Alzubaidi N, Ousman Y, Bailey M, Chris Z, Castillo-Catoni M, Fozdar P, Ramirez M, Husain M, Hudson E, Schneider I, Golabi P, Nader F. Identification of high-risk patients with nonalcoholic fatty liver disease using non-invasive tests from primary care and endocrinology real-world practices. Clin Transl Gastroenterol. 2021;12:e00340.

38. Jafarov F, Kaya E, Bakir A, Eren F, Yilmaz Y. The diagnostic utility of fibrosis- 4 or non-alcoholic fatty liver disease fibrosis score combined with liver stiffness measurement by fibroscan in assessment of advanced liver fibrosis: a biopsy-proven nonalcoholic fatty liver disease study. Eur J Gastroenterol Hepatol. 2020;32:642-649.

39. Petta $\mathrm{S}$, Wong VW, Cammà C, Hiriart JB, Wong GL, Vergniol J et al. Serial combination of non-invasive tools improves the diagnostic accuracy of severe liver fibrosis in patients with NAFLD. Aliment Pharmacol Ther 2017;46:617-627.

40. Newsome PN, Sasso M, Deeks JJ, Paredes A, Boursier J, Chan WK, Yilmaz Y, Czernichow S, Zheng MH, Wong VW, Allison M, Tsochatzis E, Anstee QM, Sheridan DA, Eddowes PJ, Guha IN, Cobbold JF, Paradis V, Bedossa P, Miette V, Fournier-Poizat C, Sandrin L, Harrison SA. FibroScan-AST (FAST) score for the non-invasive identification of patients with non-alcoholic steatohepatitis with significant activity and fibrosis: a prospective derivation and global validation study. Lancet Gastroenterol Hepatol. 2020;5:362-373.

41. Chen J, Yin M, Talwalkar JA et al. Diagnostic performance of MR elastography and vibration-controlled transient elastography in the detection of hepatic fibrosis in patients with severe to morbid obesity. Radiology 2017;283:418-428.

42. Yin M, Glaser KJ, Talwalkar JA et al. Hepatic MR elastography: clinical performance in a series of 1377 consecutive examinations. Radiology 2016;278:114-124.

43. Younossi ZM, Noureddin M, Bernstein D, Kwo P, Russo M, Shiffman ML, Younes Z, Abdelmalek M. Role of non-invasive tests in clinical gastroenterology practices to identify patients with nonalcoholic steatohepatitis at high risk of adverse outcomes: expert panel recommendations. Am J Gastroenterol. 2021;116:254-262.

44. Prabhakar O, Bhuvaneswari M. Role of diet and lifestyle modification in the management of non-alcoholic fatty liver disease and type 2 diabetes. Tzu Chi Med J. 2020;33:135-145.

45. Brunner KT, Henneberg CJ, Wilechansky RM, Long MT. Nonalcoholic fatty liver disease and obesity treatment. Curr Obes Rep. 2019:8:220-228.

46. Zaharia OP, Kupriyanova Y, Karusheva Y, et al. Improving insulin sensitivity, liver steatosis and fibrosis in type 2 diabetes by a foodbased digital education-assisted lifestyle intervention program: a feasibility study. Eur J Nutr. 2021. https://doi.org/10.1007/ s00394-021-02521-3.

47. Malespin MH, Barritt AS 4th, Watkins SE, Schoen C, Tincopa MA, Corbin KD, Mospan AR, Munoz B, Trinh HN, Weiss LM, Reddy KR, Loomba R, Kemmer N, Lok AS. Weight loss and weight regain in usual clinical practice: results from the TARGET-NASH observational cohort. Clin Gastroenterol Hepatol. 2021;S1542-3565:00073-00082.

48. Sumida Y, Yoneda M. Current and future pharmacological therapies for NAFLD/NASH. J Gastroenterol. 2018;53:362-376.

49. Cosentino F, Grant PJ, Aboyans V et al. 2019 ESC Guidelines on diabetes, pre-diabetes, and cardiovascular diseases developed in collaboration with the EASD. Eur Heart J. 2020;41:255-323.

50. Iranshahy M, Rezaee R, Karimi G. Hepatoprotective activity of metformin: a new mission for an old drug? Eur J Pharmacol. 2019;5:1-7.

51. Kasmari AJ, Welch A, Liu G, Leslie D, McGarrity T, Riley T. Independent of cirrhosis, hepatocellular carcinoma risk is increased with diabetes and metabolic syndrome. Am J Med. 2017;130:746.e1-746.e7. 
52. Said A, Akhter A. Meta-analysis of randomized controlled trials of pharmacologic agents in non-alcoholic steatohepatitis. Ann Hepatol. 2017; 16:538-547.

53. Drucker DJ. Mechanisms of action and therapeutic application of glucagon-like peptide-1. Cell Metab. 2018;27:740-756.

54. Zhang Y, Cai T, Zhao J, Guo C, Yao J, Gao P, Dong J, Liao L. Effects and safety of sitagliptin in non-alcoholic fatty liver disease: a systematic review and meta-analysis. Horm Metab Res. 2020;52:517-526.

55. Alam S, Ghosh J, Mustafa K, Ahmad N. Effect of sitagliptin on hepatic histological activity and fibrosis of non-alcoholic steatohepatitis patients: a 1-year randomized control trial. Hepat Med. 2018;10:23-31.

56. Joy TR, McKenzie CA, Tirona RG, Summers K, Seney S, Chakrabarti S, Malhotra N, Beaton MD. Sitagliptin in patients with nonalcoholic steatohepatitis: a randomized, placebo-controlled trial. World J Gastroenterol. 2017;23:141-150.

57. Bonnet F. GLP-1 receptor agonist confer target organ protection in type 2 diabetes. Diabetes Metab. 2017;43 Suppl 1:2S1-2S2.

58. Mantovani A, Petracca G, Beatrice G, Csermely A, Lonardo A, Targher G. Glucagon-like peptide-1 receptor agonists for treatment of nonalcoholic fatty liver disease and nonalcoholic steatohepatitis: an updated meta-analysis of randomized controlled trials. Metabolites. 2021;11:73.

59. Armstrong MJ, Gaunt P, Aithal GP et al. Liraglutide safety and efficacy in patients with non-alcoholic steatohepatitis (LEAN): a multicentre, double-blind, randomised, placebo-controlled phase 2 study. Lancet. 2016;387:679-690.

60. Newsome PN, Buchholtz K, Cusi K, Linder M, Okanoue T, Ratziu V, Sanyal AJ, Sejling AS, Harrison SA; NN9931-4296 Investigators. A placebo-controlled trial of subcutaneous semaglutide in nonalcoholic steatohepatitis. N Engl J Med. 2021;384:1113-24.

61. Shao N, Kuang HY, Hao M, Gao XY, Lin WJ, Zou W. Benefits of exenatide on obesity and non-alcoholic fatty liver disease with elevated liver enzymes in patients with type 2 diabetes. Diabetes Metab Res Rev. 2014;30:521-529.

62. Gastaldelli A, Repetto E, Guja C, Hardy E, Han J, Jabbour SA, Ferrannini E. Exenatide and dapagliflozin combination improves markers of liver steatosis and fibrosis in patients with type 2 diabetes. Diabetes Obes Metab. 2020;22:393-403.

63. Koepsell H. The Na+-D-glucose cotransporters SGLT1 and SGLT2 are targets for the treatment of diabetes and cancer. Pharmacol Ther. 2017;170:148-165.

64. Kahl S, Gancheva S, Straßburger K, Herder C, Machann J, Katsuyama H. Empagliflozin effectively lowers liver fat content in well-controlled type 2 diabetes: a randomized, double-blind, phase 4, placebo-controlled trial. Diabetes Care. 2020;43:298-305.

65. Latva-Rasku A, Honka M-J, Kullberg J, Mononen N, Lehtimäki T, Saltevo J. The SGLT2 inhibitor dapagliflozin reduces liver fat but does not affect tissue insulin sensitivity: a randomized, doubleblind, placebo-controlled study with 8-week treatment in type 2 diabetes patients. Diabetes Care. 2019;42:931-937.

66. Arai T, Atsukawa M, Tsubota A, Mikami S, Ono H, Kawano T, Yoshida Y, Tanabe T, Okubo T, Hayama K, Nakagawa-Iwashita A, Itokawa N, Kondo C, Kaneko K, Emoto N, Nagao M, Inagaki K, Fukuda I, Sugihara H, Iwakiri K. Effect of sodium-glucose cotransporter 2 inhibitor in patients with non-alcoholic fatty liver disease and type 2 diabetes mellitus: a propensity scorematched analysis of real-world data. Ther Adv Endocrinol Metab. 2021;21:20420188211000244.

67. Zelniker TA, Wiviott SD, Raz I, Im K, Goodrich EL, Furtado RHM. Comparison of the effects of glucagon-like peptide receptor agonists and sodium-glucose cotransporter 2 inhibitors for prevention of major adverse cardiovascular and renal outcomes in type 2 diabetes mellitus. Circulation. 2019;139:2022-2031.

68. Zelniker TA, Wiviott SD, Raz I, Im K, Goodrich EL, Bonaca MP. SGLT2 inhibitors for primary and secondary prevention of cardiovascular and renal outcomes in type 2 diabetes: a systematic review and meta-analysis of cardiovascular outcome trials. Lancet Lond Engl. 2019;393:31-39.

69. Wong C, Yaow CYL, Ng CH, Chin YH, Low YF, Lim AYL, Muthiah MD, Khoo CM. Sodium-glucose co-transporter 2 inhibitors for non-alcoholic fatty liver disease in asian patients with type 2 diabetes: a meta-analysis. Front Endocrinol (Lausanne). 2021;11:609135.

70. Kuchay MS, Krishan S, Mishra SK, Farooqui KJ, Singh MK, Wasir JS, Bansal B, Kaur P, Jevalikar G, Gill HK, Choudhary NS, Mithal A. Effect of empagliflozin on liver fat in patients with type 2 diabetes and non-alcoholic fatty liver disease: a randomized controlled trial (E-LIFT trial). Diabetes Care. 2018;41:1801-1808.

71. Latva-Rasku A, Honka MJ, Kullberg J, Mononen N, Lehtimäki T, Saltevo J, Kirjavainen AK, Saunavaara V, Iozzo P, Johansson L, Oscarsson J, Hannukainen JC, Nuutila P. The SGLT2 inhibitor dapagliflozin reduces liver fat but does not affect tissue insulin sensitivity: a randomized, double-blind, placebo-controlled study with 8 -week treatment in type 2 diabetes patients. Diabetes Care. 2019;42:931-937.

72. Lai L-L, Vethakkan SR, Nik Mustapha NR, Mahadeva S, Chan W-K. Empagliflozin for the treatment of non-alcoholic steatohepatitis in patients with type 2 diabetes mellitus. Dig Dis Sci. 2020;65:623-631. https://doi.org/10.1007/s10620-019-5477-1.

73. Bougarne N, Weyers B, Desmet SJ, Deckers J, Ray DW, Staels B, De Bosscher K. Molecular actions of PPAR $\alpha$ in lipid metabolism and inflammation. Endocr Rev. 2018;39:760-802.

74. Cusi K, Orsak B, Bril F, Lomonaco R, Hecht J, Ortiz-Lopez C, Tio F, Hardies J, Darland C, Musi N, Webb A, Portillo-Sanchez P. Long-term pioglitazone treatment for patients with nonalcoholic steatohepatitis and prediabetes or type 2 diabetes mellitus: a randomized trial. Ann Intern Med. 2016;165:305-315.

75. Musso G, Cassader M, Paschetta E, Gambino R. Thiazolidinediones and advanced liver fibrosis in nonalcoholic steatohepatitis: a meta-analysis. JAMA Intern Med. 2017;177:633-640.

76. Zhou Y, Huang Y, Ji X, Wang X, Shen L, Wang Y. Pioglitazone for the primary and secondary prevention of cardiovascular and renal outcomes in patients with or at high risk of type 2 diabetes mellitus: a meta-analysis. J Clin Endocrinol Metab. 2020;105:dgz252.

77. Sumida Y, Seko Y, Yoneda M; Japan Study Group of NAFLD (JSG-NAFLD). Novel anti-diabetic medications for non-alcoholic fatty liver disease with type 2 diabetes mellitus. Hepatol Res. 2017:47:266-80.

78. Gawrieh S, Noureddin M, Loo N, et al. Saroglitazar, a PPAR- $\alpha / \gamma$ agonist, for treatment of nonalcoholic fatty liver disease: a randomized controlled double-blind phase 2 trial. Hepatology. 2021. https://doi.org/10.1002/hep.31843.

Publisher's Note Springer Nature remains neutral with regard to jurisdictional claims in published maps and institutional affiliations. 